\title{
Das Scheitern der Politikwissenschaft am Bündnis für Arbeit. Eine Kritik an der Problemlösungsliteratur über das Bündnis für Arbeit*
}

\author{
Christine Trampusch
}

Der Beitrag stellt das Bündnis für Arbeit der Literatur über das Bündnis für Arbeit gegenüber. Als gemeinsamer Nenner der Literatur lässt sich ihre Neigung definieren, problemlösungsorientiert zu sein. Sie erwartete vom Bündnis für Arbeit eine kollektive Lösung der Probleme auf dem Arbeitsmarkt. Aus der Betrachtung der Entstehung des Bündnisses und des Konfliktes um die Rente mit 60 plädiert das Papier dafür, das Verhalten und die Handlungen der Bündnisakteure weniger anhand ihrer Fähigkeit zur Produktion einer kollektiven Problemlösung zu analysieren und damit auch den Selbststilisierungen der Bundesregierung zu unterliegen, als auf der Grundlage der macht- und interessenpolitischen Auseinandersetzungen zwischen den Bündnisakteuren.

\section{Einleitung}

In demokratischen Gesellschaften wird in der Öffentlichkeit von den Politikern erwartet, dass sie „Probleme lösen“. Politiker gehen auf diese Erwartung ein, indem sie sich aus wahlpolitischen Gründen bemühen, ihre Fähigkeit zur Problemlösung zu zeigen. Die meisten von ihnen „wissen“ freilich, dass es in der Politik nicht um Problemlösungen geht, sondern um Politik, also um das "Streben nach Machtanteil oder nach Beeinflussung der Machtverteilung [...] innerhalb des Staates zwischen den Menschgruppen, die er umschließt" (Weber 1992 [1919]: 7). Die Politikwissenschaft weiß das auch. Trotzdem spielt sie oft naiv: Sie nimmt Forderungen der "Öffentlichkeit" und die Selbststilisierungen der Politiker für bare Münze, erwartet „Problemlösungen“ und analysiert und bewertet das Verhalten und die Handlungen der Politiker danach, ob sie Probleme lösen.

Das Bündnis für Arbeit und die politikwissenschaftliche Literatur über das Bündnis zeigen diese Spannung zwischen der Selbststilisierung der Politiker als Problemlöser, einer Politikwissenschaft, die dieser Selbststilisierung verfällt, und der Politik. Die Bundesregierung hat angekündigt, durch das Bündnis im Konsens und mit Hilfe der Sozialpartner die Probleme auf dem Arbeitsmarkt zu lösen. Dies hat die Politikwissenschaft vom Bündnis für Arbeit auch erwartet, sehr schnell aber kläglich vermisst und das Bündnis dann, lange bevor es die Bundesregierung im März 2003 auflöste, für gescheitert erklärt. Betrachtet man jedoch Entstehungsgeschichte und Verlauf des Bündnisses, so spricht vieles dafür, dass die Analyse des Bündnisprozesses aus der Perspektive

* Für hilfreiche Kritik danke ich Anke Hassel, Armin Schäfer, Wolfgang Streeck sowie den beiden anonymen Gutachtern der PVS. 
der problemorientierten Politikwissenschaft dem Gegenstand nicht angemessen ist. Die Entstehung des Bündnisses und die Ziele der Bündnisakteure waren nicht problemorientiert, sondern interessen- und machtpolitisch bedingt.

Die Entstehungsgeschichte des Bündnisses zeigt, dass sowohl die Sozialdemokratie als auch Arbeitgeber und Gewerkschaften ein substanzielles Interesse am Bündnis entwickelten, jedoch nicht, weil die Problemkonstellation des Arbeitsmarktes und Sozialstaates dazu geraten hätte. Warum es zum Bündnis kam, erklärt sich vielmehr aus machtpolitischen Gründen: Die Bündnisakteure verfolgten mit und im Bündnis Partialinteressen organisatorischer und verteilungspolitischer Art, statt sich der Lösung „allgemeiner" Probleme zuzuwenden. Die Sozialdemokraten instrumentalisierten den Bündnisgedanken für die Bundestagswahlen 1998. Für sie war das Bündnis für Arbeit zentraler Bestandteil der Politikwechselkampagne, die sie gemeinsam mit den Gewerkschaften zur Ablösung der Regierung Kohl initialisiert und durchgeführt hatten. Die Gewerkschaften sahen das Bündnis als Instrument an, ihrer seit Mitte der 1990er Jahre ins Straucheln geratenen privilegierten Stellung in der sozialpolitischen Politikgestaltung neuen Rückhalt zu verschaffen. Die Arbeitgeber wiederum wollten durch ihre Teilnahme am Bündnis das Zusammenspiel von Gewerkschaften und Sozialdemokraten kontrollieren. Im Kontext der folgenden tarif- und sozialpolitischen Auseinandersetzungen war es im weiteren Verlauf des Bündnisses jedoch vor allem die IG Metall, die vom Bündnis Gebrauch machte. Sie wollte Regierung und Arbeitgeber durch das Bündnis auf die Rente mit 60 verpflichten. Die IG Metall scheiterte damit, was letztendlich den Anfang vom Ende der alten IG Metall einleitete.

Meine These ist, dass für die Beantwortung der Fragen, warum das Bündnis gegründet wurde und welche Ziele mit und in dem Bündnis von Bundesregierung, Gewerkschaften und Arbeitgeberverbänden verfolgt wurden, eine problemorientierte Perspektive wenig hilfreich ist. Stattdessen sollte eine Perspektive eingenommen werden, die das Bündnis als Opportunitätsstruktur für alte und während des Bündnisprozesses noch zu entdeckende Ziele betrachtet. Im Bündnis für Arbeit standen Machterhaltungs-, Machtverschiebungs- und Machtverteilungsinteressen im Vordergrund.

Dieses Papier gliedert sich in drei Abschnitte. Der erste Abschnitt greift die problemorientierte Politikwissenschaft auf und zeigt, wie Politik dort analysiert wird. Daran anschließend wird dargestellt, dass die Bündnisliteratur das Bündnis für Arbeit und seine Akteure als problemorientiert kommuniziert hat. Der zweite Abschnitt rekonstruiert die Entstehung des Bündnisses und dessen Verlauf am Beispiel des Konfliktes um die "Rente mit 60". Auf Grundlage einer empirischen Analyse des Bündnisprozesses fragt er nach den Handlungsorientierungen der Bündnisakteure. Der dritte Abschnitt konfrontiert resümierend die Empirie des Bündnisses mit dem politikwissenschaftlichen Diskurs über das Bündnis. Dabei wird deutlich: Die Literatur über das Bündnis für Arbeit hat eine Wirklichkeit konstruiert, in der die Bündnisakteure nicht gelebt haben. Sie ist deshalb bei der Analyse des Bündnisprozesses gescheitert. 


\section{Die Bündnisliteratur als problemorientierte Politikwissenschaft}

Problemorientierung ist in der deutschen Politikwissenschaft fest etabliert. Die Einführung des Begriffs „Policies“ und Lowis Erkenntnis „policies determine politics“ (Lowi 1972: 299) haben maßgeblich dazu beigetragen. Problemorientierte Politikwissenschaft analysiert politische Entscheidungen unter der Maßgabe, dass Policies eine erklärende Variable darstellen (vgl. dazu Benz 1997). Sie (unter)sucht Problemlösungen. Mit Benz (1997: 306) gesprochen gehen die Policy-Studien der problemorientierten Politikwissenschaft davon aus, dass „definierte Policies Strategien und Entscheidungen der politischen Akteure sowohl hinsichtlich sachlicher Themen wie auch hinsichtlich der Entscheidungsstrukturen beeinflussen. “Zwei Prämissen werden gesetzt: 1.) Politische Entscheidungen stellen inhaltliche Problemdefinitionen dar: „[D] urch die Definition einer Policy wird ein Problem entscheidungsfähig und damit zu einer ,Aufgabe“ von Politik“ (Benz 1997: 310). 2.) Politische Interaktionen finden in einem von der Policy vorgegebenen und stabilen institutionellen Rahmen statt, innerhalb dessen Problembearbeiter strategisch und interdependent agieren. Die zwei Prämissen haben zur Folge, dass politische Entscheidungen auf der Grundlage eines Modells effektiver Problemlösungspolitik untersucht werden. Problemdefinitionen, Interessen und Handlungsstrategien der Akteure sowie institutionelle Strukturen sind zwar keineswegs vorbestimmt, weil Akteure „im modernen Regierungssystem unterschiedliche Zugänge der Interessenvermittlung in Regierung, Parlament, Parteien und Verwaltungen nutzen“ (Benz 1997: 305) können. Akteure werden jedoch ,jene Strukturen wählen, die ihren Interessen und dem zu lösenden Problem am besten angemessen sind“ (Benz 1997: 305; Hervorhebung nicht im Original).

In der analytischen Narration von politischen Entscheidungen bzw. Prozessen geht die problemorientierte Politikwissenschaft daher folgendermaßen vor: Im ersten Schritt suchen sich Policy-Studien ein spezielles gesellschaftliches Problem (z.B. Umweltschutz, Europäische Integration, Terrorismus, Arbeitslosigkeit) und bestimmen Akteure und Institutionen, die bei der Bearbeitung des Problems aktiv beteiligt sind und/oder die Problembearbeiter bewusst oder unbewusst, intendiert oder nicht intendiert, manifest oder latent beeinflussen. Im zweiten Schritt wird der politische Entscheidungsprozess vor dem Hintergrund der institutionellen Handlungsbeschränkungen und Eigeninteressen der Akteure als Prozess der Suche nach machbaren Problemlösungen modelliert und untersucht. Im dritten Schritt wird schließlich das Ergebnis des Interaktionsprozesses, die politische Entscheidung, bewertet. Dabei zeigen sich vornehmlich zwei Vorgehensweisen: Es wird gefragt, ob sich Problemlösungen geändert haben, und/oder es werden die Wirkungen der politischen Maßnahmen auf das Problem untersucht. Drei Faktoren bestimmen die Durchführbarkeit von Problemlösungen bzw. können effektive Problemlösungspolitik verhindern: 1.) Problemdefinitionen, weil diese „inhaltliche Beschränkungen für die Strategiewahlen der Akteure“ (Benz 1997: 310) setzen, 2.) institutionelle und organisationsstrukturelle Handlungsbeschränkungen sowie 3.) die Eigeninteressen der Akteure.

Die Heuristik der problemorientierten Politikwissenschaft berücksichtigt damit durchaus interessen- und machtpolitische Kalküle der Akteure. Welche gesellschaftlichen Probleme Parteien als zu bearbeitende Probleme definieren und wie sie diese aus- 
legen, ist nicht ganz unabhängig von partei- und wahlpolitischen Strategien. Auch bei der Bearbeitung des Problems und der Formulierung einer politischen Maßnahme kommen partei- oder verbandspolitische Eigeninteressen zum Tragen. Die Eigeninteressen der Akteure stellen in der problemorientierten Politikwissenschaft jedoch nicht mehr als eine erklärende Variable für abweichendes Verhalten von effektiver Problemlösungspolitik dar. ${ }^{1}$ Weil eine politische Partei wieder gewählt werden will, vermeidet sie während des Wahlkampfes einschneidende sozialpolitische Kürzungen, auch wenn dies langfristig von ökonomischen Analysen als beschäftigungsfördernd betrachtet wird. Eine Gewerkschaft, deren Mitgliederstamm die über 40-Jährigen darstellt, wird nicht aktiv bei der Reform der Frühverrentungspolitik mitarbeiten, auch wenn die Erhöhung der Aktivitätsrate als conditio sine qua non zur Verbesserung der Arbeitsmarktperformanz gilt.

Auf den ersten Blick stellt das Bündnis für Arbeit einen Gegenstand dar, auf den die Prämissen der problemorientierten Politikwissenschaft zutreffen. 1998 erklärte die neue (designierte) Bundesregierung, mit dem Bündnis für Arbeit gesellschaftliche Probleme lösen zu wollen: Die Problemlösung auf dem Arbeitsmarkt wurde zur Aufgabe der Politik erklärt. Und genau für diese Aufgabe wurde ein Bündnis für Arbeit als notwendig erachtet. So versprach der erste Bundeskanzleramtsminister der rot-grünen Bundesregierung Bodo Hombach in seiner Beschreibung der "Politik der Neuen Mitte“ 1998 eine „neue ,konzertierte Aktion' zur Lösung der drängenden Probleme“ (Hombach 1998: 118). Und der SPD-Kanzlerkandidat Gerhard Schröder umschrieb auf dem SPD-Parteitag, der im April 1998 das Wahlprogramm beschloss, das Bündnis für Arbeit folgendermaßen: „Wie alle neuen Maßnahmen werden wir die Rücknahmen und Korrekturen in der Konsensrunde des neuen ,Bündnisses für Arbeit' abgleichen. Wir werden ein Gesamtkonzept, mit den Kolleginnen und Kollegen in den Gewerkschaften, mit den Arbeitgebern, aber auch zusammen mit der Wissenschaft schmieden, ein Konzept das Sozialpolitik und Arbeitsmarktpolitik, Steuerpolitik und Wirtschaftspolitik miteinander verzahnt und auf diese Weise ein gesellschaftliches Klima des Vertrauens schafft, in dem wirtschaftlicher Aufschwung nicht nur als Chance, sondern als Realität wieder möglich wird“ (zit. nach Müller/Wilke 1999: 111).

Die Politikwissenschaft nahm diese Ankündigungen ernst. Dem Bündnis für Arbeit wurde in der politikwissenschaftlichen Literatur nicht nur große Aufmerksamkeit zuteil, es wurde auch in die Tradition der Konzertierten Aktion (Esser/Schroeder 1999:

1 So konstatiert Benz (1997: 313): „Politische Entscheidungsprozesse werden durch Akteure vorangetrieben, die eigene Strategien und Interessen verfolgen. Durch die Definition von Policies setzten sie sich einen Handlungsrahmen, der ihre Strategien und die Art ihrer Interessenverfolgung beeinflußt. Diese Akteure sind aber normalerweise Vertreter von Organisationen, seien es Regierungs- bzw. Verwaltungsorganisationen, Parteien, Verbände oder Unternehmen. Als Beteiligte in politischen Prozessen zwischen diesen Organisationen unterliegen sie den inhaltlichen Prämissen, die durch die Definition von Politiken gesetzt sind, als Angehörige von Organisationen sind sie jedoch an Erwartungen und Handlungsbeschränkungen gebunden, die innerhalb der organisatorischen Arena gelten [...] Diese Tatsache bewirkt zunächst, daß in interorganisatorischen politischen Prozessen unter Umständen neben den von den Beteiligten anerkannten Policy-Definitionen davon abweichende Problem- und Zieldefinitionen zum Tragen kommen. Wenn sich im politischen Entscheidungsprozeß eine Policy-Definition herausgebildet hat, muß dies nicht bedeuten, daß diese auch den Interpretations- und Orientierungsrahmen für die innerorganisatorischen Prozesse bildet." 
11; Schroeder 2001: 49)² gestellt und als „ein aktueller Versuch“ definiert, „das in unserem historisch gewachsenen Verbändewesen akkumulierte soziale Kapital zur Aushandlung grundlegender Reformen nutzbar zu machen" (Streeck 1999a: 4). Eichhorst (2002) erwartete vom Bündnis auch die Nachahmung des niederländischen Beschäftigungswunders.

Die Grundlage für die folgende Literaturanalyse bilden 42 Artikel, die zwischen 1998 und 2003 publiziert wurden, und die das Bündnis für Arbeit zum Hauptgegenstand hatten oder zumindest an prominenter Stelle behandelten. Dabei handelt es sich nicht nur um politikwissenschaftliche Beiträge (Behrens/Fichter/Frege 2001; Braunthal 1999; Dyson 2001; Eichhorst 2002; Esser/Schroeder 199933 Hassel 1999, 2000, 2001, 2002; Jochem/Siegel 1999, 2003; Leggewie 1999; Mätzke 2000; Schroeder 2001, 2003; Schroeder/Esser 1999; Siegel 2003; Silvia 1999, 2002; Streeck 1999a, 1999b, 2001, 2003a, 2003b), sondern auch um Beiträge von Mitarbeitern gewerkschaftlicher Einrichtungen (Bispinck/Schulten 1999, 2000), Verbandsfunktionären (Arlt/Nehls 1999; Haß/Küntzel 2003; Hensche 1999a, 1999b; Lang 2001a, 2001b, 2002; Schmitthenner 1998; Schulte 2000, 2002) und gewerkschaftsnaher Publizisten (Creutz 1999; Detje/König 19994). ${ }^{5}$

Betrachtet man die Beiträge über das Bündnis für Arbeit systematisch, so zeigt sich, dass sie Entstehung und Verlauf des Bündnisses auf Grundlage der drei analytischen Schritte der problemorientierten Politikwissenschaft beschreiben: Ausgehend von gesellschaftlichen Problemen auf dem Arbeitsmarkt wird das Bündnis für Arbeit als Institution definiert, das Bundesregierung, Gewerkschaften und Arbeitgeberverbände bei der Lösung dieser Probleme beeinflusst (erster Schritt: Problemlösungsinstitution Bündnis). Der Bündnisprozess und die Bündnisentscheidungen werden sodann vorrangig unter der Perspektive betrachtet, inwieweit es den Akteuren gelang, im Bündnis für Arbeit diese Problemlösungen auch zu formulieren und zu beschließen (zweiter Schritt: Suche nach machbaren Problemlösungen). Abschließend wird das Bündnis als Problemlösungsinstitution bewertet (dritter Schritt: Bewertung der Problemlösungsinstitution Bündnis). Hinsichtlich dieser Vorgehensweise besteht dabei zwischen den politikwissenschaftlichen und den nicht politikwissenschaftlichen Beiträgen wenig Unterschied. Beide definieren und bewerten das Bündnis als Problemlösungsinstitution. Der Frage nach machbaren Problemlösungen wird hingegen - mit Ausnahme der Beiträge von Schmitthenner (1998), Haß/Küntzel (2003) und Hensche (1999a, 1999b) - vorrangig in den politikwissenschaftlichen Beiträgen nachgegangen.

2 Gleichwohl wurden auch die Unterschiede zwischen der Konzertierten Aktion und dem Bündnis für Arbeit betont, die in den Rahmenbedingungen, Problemen und Konzepten gesehen wurden (Schroeder 2001: 50).

3 Wolfgang Schroeder ist auch beim Vorstand der IG Metall tätig.

4 Richard Detje ist Redakteur der Zeitschrift „Sozialismus“. Otto König ist 1. Bevollmächtigter der Verwaltungsstelle Gevelsberg-Hattingen und Mitglied im Vorstand der IG Metall.

5 Die Beiträge des Soziologen Heinze (2003), des Juristen Volker Rieble (1999), des Ökonomen Wolfgang Franz (2000) und von Janina Scheelhaase (2001), die bei der Prognos AG tätig ist, lassen sich in die oben genannte Einteilung nicht einfügen. 
2.1 Erster Schritt: Problemlösungsinstitution Bündnis

Mit wenigen Ausnahmen analysiert die Literatur das Bündnis auf der Basis gesellschaftlicher Probleme, deren Lösung sie sich von dieser Institution versprach. Zwei Hauptprobleme werden dabei genannt: das Arbeitsmarktproblem und das Konsensproblem. In der Folge lässt sich als ein gemeinsamer Nenner einer Vielzahl von Beiträgen feststellen, dass sie vom Bündnis eine korporatistische Problemlösung erwarteten. So sollte das Bündnis durch einen im Konsens gestalteten Wandel bei der Formulierung und Durchsetzung von Reformen und Maßnahmen dienlich sein, die

- die Gewerkschaften auf eine beschäftigungsfördernde Tarifpolitik verpflichten (so insbesondere Hassel 2000, 2001, 2002),

- die Arbeitslosigkeit bekämpfen (so insbesondere Arlt/Nehls 1999; Hensche 1999a; Jochem/Siegel 1999; Lang 2001a; Leggewie 1999; Rieble 1999; Schmitthenner 1998; Siegel 2003; Silvia 1999) oder

- Beschäftigung fördern (insbesondere Bispinck/Schulten 2000; Braunthal 1999; Hensche 1999b; Lang 2001a; Rieble 1999; Schmitthenner 1998; Silvia 2002).

Bei der Interpretation des Bündnisses als einen korporatistischen Problemlösungsversuch bezieht sich die Literatur dabei entweder - jedoch meist implizit - auf die korporatistische Diskussion über Steuerungs-Korporatismus (liberal corporatism, Lehmbruch 1977) und Korporatismus als Konzertierung (corporatist concertation, Lehmbruch 1984) oder sie verweist auf die bereits erwähnten Ankündigungen der Bundesregierung. Während das Bündnis als Steuerungs-Korporatismus ${ }^{6}$ bei der Formulierung und Implementation von Problemlösungen auf dem Arbeitsmarkt und der Reform der sozialen Sicherungssysteme dienlich sein sollte, wäre die Funktion des Bündnisses als Konzertierung ${ }^{7}$ gewesen, dass sich die Bündnisakteure auf dreiseitige Vereinbarungen einigen, die Lohnpolitik und Sozialpolitik im gesamtwirtschaftlichen Interesse ausgestalten und Lohnzurückhaltung der Gewerkschaften erwirken (sozialer Pakt, Beschäftigungspakt, verhandelte Anpassung). ${ }^{8}$ Hätte das Bündnis als Steuerungs-Korporatismus eine Konsensbildung herbeiführen sollen, die die Selbstregulierungskompetenz von Ar-

6 Steuerungs-Korporatismus definiert Lehmbruch (1977: 94) als ein „institutionalized pattern of policy-formulation in which large interest organizations cooperate with each other and with public authorities not only in the articulation (or even ,intermediation') of interests, but - in its developed forms - in the ,authoritative allocation of values' and in the implementation of such policies".

7 Korporatismus als „Konzertierung“ definiert Lehmbruch (1984: 62) in Abgrenzung zu „sektoralem Korporatismus“: „,Corporatist concertation“ is distinguished from sectoral corporatism by two essential features: (i) it involves not just a single organized interest with privileged access to government but rather a plurality of organizations usually representing antagonist interests; and (ii) these organizations manage their conflicts and co-ordinate their action with that of government expressly in regard to the systemic (gesamtwirtschaftliche) requirements of the national economy" (Hervorhebung im Original).

8 Als makrokorporatistische Konzertierung (Sozialer Pakt, Beschäftigungspakt) wurde das Bündnis in den folgenden Beiträgen definiert: Bispinck/Schulten 2000; Detje/König 1999; Dyson 2001; Eichhorst 2002; Esser/Schroeder 1999; Hassel 2000, 2001, 2002; Haß/Küntzel 2003; Hensche 1999a; Jochem/Siegel 1999; Lang 2001b; Lehmbruch 2000; Mätzke 2000; Schroeder 2001, 2003; Schroeder/Esser 1999; Schulte 2000 (implizit); Siegel 2003; Streeck 1999b, 2001, 2003a (implizit). 
beitgebern und Gewerkschaften für eine outputorientierte Staatsentlastung nutzt, so hätte eine Konzertierung vom Bündnis politikfeldübergreifende Absprachen und damit die Regulierung von Verteilungskonflikten zwischen Politikbereichen verlangt.

Der inflationäre Gebrauch der Begriffe „makrokorporatistische Konzertierung“, „sozialer Pakt" oder „Beschäftigungspakt" lässt sich sicherlich darauf zurückführen, dass die Bundesregierung das Bündnis als ein solches Ereignis definierte. ${ }^{9}$ Des Weiteren folgte diese Etikettierung aber auch einem aktuellen Trend in der Staat-Verbände-Forschung. Das Bündnis für Arbeit entstand nämlich gerade zu jener Zeit, als sich dort eine Debatte über soziale Pakte und neue Einkommenspolitik ausbreitete (Ebbinghaus/ Hassel 1999; Fajertag/Pochet 1997; Hassel 1999, 2003; Martin 1997). Das Bündnis wurde dann sehr schnell in diesen Bezugsrahmen eingeordnet (vgl. z.B. Ebbinghaus/ Hassel 1999; Jochem/Siegel 2003). Konzertierungsergebnisse der Beschäftigungspakte in den anderen EU-Mitgliedsländern wurden als Messlatte der Bewertung des Bündnisses verwandt (z.B. Siegel 2003). ${ }^{10}$

\subsection{Zweiter Schritt: Machbare Problemlösungen}

Die Bündnisliteratur vollzieht bei der Analyse des Bündnisprozesses auch den zweiten Schritt der problemorientierten Politikwissenschaft. Die Bündnisentscheidungen werden vor dem Hintergrund der Handlungsbeschränkungen und Eigeninteressen der Akteure als - wenn auch erfolglose - Suche nach machbaren Problemlösungen untersucht. Deutlich wird dies, wenn man in den Blick nimmt, welche Ursachen für das Scheitern des Bündnisses als korporatistische Problemlösungsstrategie (Steuerungsinstrument) genannt werden. Hier werden vornehmlich solche Faktoren angeführt, die darauf verweisen, dass für eine makrokorporatistische Problemlösung institutionelle und organisationsstrukturelle Hindernisse bestanden. Daneben verhinderten Eigeninteressen der Akteure sowie dem Problem unangemessene Problemdefinitionen eine kollektive Problemlösung. Die Bündnisakteure konnten und wollten keine Konzertierung betreiben.

So konstatieren viele Beiträge, dass das Bündnis an institutionellen Hindernissen scheiterte: In der Bundesrepublik stehen der Aushandlung eines politischen Tausches aufgrund der institutionellen Segmentierung des Wohlfahrtsstaates und der institutionellen Blockade durch Parteienwettbewerb und Verhandlungsdemokratie Blockaden entgegen (als erster Lehmbruch 2000). Heinze (2003), Jochem/Siegel (1999) und Sie-

91999 beschrieb Bundeskanzler Gerhard Schröder das Bündnis für Arbeit folgendermaßen: „Die Begriffe sind zwar verschieden, doch ob Bündnis für Arbeit, Konzertierte Aktion, Sozialoder Beschäftigungspakt - die Idee ist in fast allen westeuropäischen Staaten dieselbe: Die Massenarbeitslosigkeit lässt sich nur dann wirkungsvoll bekämpfen, wenn sich Steuerpolitik, Sozialpolitik, Tarifpolitik und Zukunftsinvestitionen sinnvoll ergänzen. Denn erst im Zusammenwirken von Staat, Arbeitgebern und Gewerkschaften kann in einer Volkswirtschaft dauerhaft mehr Beschäftigung entstehen" (Schröder 1999: 49).

10 So stellt Siegel (2003: 171) die Frage, „warum von der korporatistischen Makrokonzertierung angesichts dringlicher sozial- und beschäftigungspolitischer Probleme nicht diejenigen Reformimpulse ausgingen, welche die Bundesregierung bei der Initiierung der dreiseitigen Konzertierung ursprünglich angestrebt hatte, und die in anderen EU-Ländern im Zuge ähnlich gelagerter Problemkonstellationen von der angebotspolitischen Konzertierung ausgelöst werden konnten". 
gel (2003), weniger explizit auch Eichhorst (2002) und Hassel $(2000$, 2002) führen das Scheitern des Bündnisses für Arbeit als Konsensrunde zur Aushandlung von politikfeldübergreifenden Austauschprozessen mit Lehmbruch auf die interne Differenzierung des Wohlfahrtsstaates zurück, die eine sektorenübergreifende Konsensbildung und Systemsteuerung an relativ autonomen sektoralisierten Arenen scheitern lässt. Siegel (2003) nennt Blockaden, die durch den Parteienwettbewerb entstanden. Als weitere Ursachen des Scheiterns des Bündnisses als Konzertierungsinstrument werden organisationsstrukturelle Hindernisse aufgeführt: Weder die Gewerkschaften noch der Staat verfügten über die für ein makrokorporatistisches Arrangement notwendige innere Verpflichtungsfähigkeit. Die IG Metall kann ihre Untergliederungen nicht kontrollieren, weshalb, so Hassel (2002: 63), die „Strategiefähigkeit der IG-Metall-Spitze [...] in sich zusammengefallen" ist (ähnlich Streeck 2003b). Der Staat unterlag Konflikten zwischen den Ressorts (Hassel 2000; Leggewie 1999; Siegel 2003; Streeck 2003b) sowie zwischen Regierung und Fraktion (Streeck 2003b) und Regierung und Partei (Streeck 2003b). Weiterhin nennt Streeck (2003b) die mangelnde Verpflichtungsfähigkeit der Arbeitgeberorganisationen als Hindernis. Und Hassel (2000) führt die Autonomie von Organisationen wie der Bundesanstalt für Arbeit als zusätzliches organisationsstrukturelles Problem des Staates an. Problemlösung scheiterte auch an Partikular- und Eigeninteressen der Akteure. Genannt werden hier Konflikte zwischen Gewerkschaften und Arbeitgeberverbänden (Schmitthenner 1998; Schroeder 2003; Silvia 2002) sowie die von den Bündnisakteuren bewusste Nichtberücksichtigung von anderen wichtigen Akteuren. ${ }^{11}$ Ideologische Unstimmigkeit zwischen der SPD und den Gewerkschaften (Lang 2002; Schroeder 2003), die fehlende ideologische Kohäsion der SPD (Leggewie 1999) und die mangelnde Agenda-Setzung durch den Bundeskanzler (Eichhorst 2002; Haß/Küntzel 2003; Hensche 1999a, 1999b; Jochem/Siegel 1999; Schroeder 2003; Siegel 2003; Silvia 2002; Streeck 2003b) verhinderten passende Problemdefinitionen.

\subsection{Dritter Schritt: Bewertung der Problemlösungsinstitution „Bündnis“}

Die Bündnisliteratur nimmt auch Bewertungen vor. Vornehmlich wird dabei nach dem Erfolg und Misserfolg des Bündnisses bei der Lösung der Beschäftigungsprobleme auf dem deutschen Arbeitsmarkt gefragt. ${ }^{12}$ Am Abbau der Arbeitslosigkeit (Verbesserung der Arbeitsmarktperformanz) bewerten Arlt/Nehls (1999), Haß/Küntzel (2003), Lang (2001b), Schmitthenner (1998), Siegel (2003), Silvia (2002) und Schroeder (2003) das Bündnis. Franz (2000), Hassel (2000, 2002), Haß/Küntzel (2003), Streeck (2001, 2003a) und Schulte (2002) beurteilen das Bündnis anhand seiner Wirkung auf Lohnzurückhaltung und eine beschäftigungsfördernde Tarifpolitik.

Für verschiedene Politikbereiche zählt die Literatur auch konkrete Maßnahmen auf, die durch das Bündnis erreicht wurden. In der Ausbildungspolitik werden das Jugend-

11 Genannt werden hier Vertreter des Geldkapitals (Creutz 1999), Arbeitslose, Schüler, Studenten, Kirchen, Sozialverbände (Hensche 1999a, 1999b) und soziales Kapital außerhalb der industriellen Interessengruppen (Leggewie 1999).

12 Scheelhaase (2001), Leiterin des Marktfeldes Makroökonomie der Prognos AG, ging gar der Frage nach, ob es „Synergieeffekte zwischen klimaschützenden Maßnahmen und dem Bündnis für Arbeit" gäbe. 
sofortprogramm "Jump“ (Bispinck/Schulten 2000) und die Steigerung der Ausbildungsplätze, neue Berufsbilder und die Intensivierung der Ausbildung im IT-Bereich (Bispinck/Schulten 2000; Eichhorst 2002; Lang 2001b; Mätzke 2000) genannt. In der Weiterbildungspolitik werden Arbeitszeitkonten (Bispinck/Schulten 2000; Eichhorst 2002; Lang 2001b) und Weiterbildungstarifverträge und -betriebsvereinbarungen (Lang 2001b) angeführt. Die Arbeitsmarktpolitik erhielt Impulse durch den 2001Bericht der Benchmarkinggruppe zur aktivierenden Arbeitsmarktpolitik (Eichhorst 2002). Die Steuerpolitik wurde durch das Bündnis bei der Ökosteuerreform, der Körperschaftsteuer- und Einkommensteuerreform, der Reform der Besteuerung der Unternehmensgewinne sowie der Haushaltskonsolidierung beeinflusst (Bispinck/Schulten 2000). Andere Autoren rechneten dem Bündnis Einigungen in der Frühverrentungspolitik (Hassel 2001; Lang 2001b) und, mit Hinblick auf die Tarifpolitik, auch den Ausgang der Tarifrunde 2000 (Bispinck/Schulten 2000; Eichhorst 2002; Haß/ Küntzel 2003; Streeck 2003a) und eine Orientierung zu einer qualitativen Tarifpolitik zu (Schulte 2002).

Neben der Thematisierung des Problemlösungserfolges gibt es auch Beiträge, die das Bündnis anhand der Veränderung des materiellen Inhalts von Politik bewerten. Nach Siegel (2003: 170) blieb Wandel gänzlich aus, weil die „durch die korporatistische Makrokonzertierung angestrebte politikfeldübergreifende, dreiseitige Koordination" nicht zustande kam. Dies heben auch Eichhorst (2002), Hassel (2002) und Heinze (2003) hervor. ${ }^{13}$ Hassel (2001: 322) und Heinze (2003: 150) bewerten das Bündnis anhand der Frage, ob „politische Austauschprozesse“ stattfanden. Schroeder/ Esser (1999) analysieren das Bündnis anhand seines Effektes auf den sozialkonsensual und politisch regulierten Kapitalismus in Deutschland. Policy-Wandel ist die Meßlatte der Beiträge von Bispinck/Schulten (1999), Hassel (2001), Heinze (2003), Lang (2001b), Silvia (1999) und Streeck (2001, 2003a). Eichhorst (2002), Haß/Küntzel (2003) und Streeck (2003a) betonen, dass die Bundesregierung das Bündnis nicht in den Dienst einer Reform der Sozialversicherung und Senkung der Lohnnebenkosten gestellt habe. Hassel (2000) und Streeck (2003b) schlussfolgern, dass die Regierung das Bündnis strategisch überhaupt nicht genutzt habe.

Unter den politikwissenschaftlichen Beiträgen gehen nur wenige Beiträge über den Analysehorizont einer problemorientierten Politikwissenschaft hinaus, indem sie beispielsweise die Stilisierung des Bündnisses als korporatistische Problemlösungsstrategie bzw. korporatistisches Steuerungsinstrument vermeiden. ${ }^{14}$ Sie tun dies, indem sie nicht gesellschaftliche Probleme zum Ausgangspunkt wählen und von diesen ausgehend den Bündnisprozess analysieren, sondern indem sie fragen, wie das Bündnis auf den politischen Prozess wirkte, weil es Interessen- und Machtauseinandersetzungen strukturierte. Damit wird - unabhängig von der Problemkonstellation auf dem Arbeitsmarkt - die Möglichkeit erwogen, dass es im Bündnis um partikulare Ziele, insbesondere auch um die Verteilung von Machtpositionen in der Politikgestaltung ging. Die interessen- und

13 Nur zwei Autoren rechnen dem Bündnis eine politikfeldübergreifende Wirkung zu: Braunthal (1999: 50) konstatiert, dass die Arbeitgeber im Bündnis für eine Reform der Körperschaftsteuer ihr prinzipielles Einverständnis für die Rente mit 60 gaben. Siegel (2003: 170) nennt die Einführung der Ökosteuer als politikfeldübergreifende Reform.

14 Unter den nicht politikwissenschaftlichen Beiträgen ist dies bei keinem der Fall. 
machtpolitischen Kalküle der Akteure im Bündnisprozess stellen dann eine $z u$ erklärende Variable dar. Dyson (2001) und Streeck (2003b) fragen, ob das Bündnis zur Steigerung der Autorität und Macht des Bundeskanzlers in Regierung, Fraktion oder Partei genutzt wurde, Mätzke (2000), ob es als strategisches Instrument im Wahlkampf 1998 eingerichtet wurde und Streeck (2003b), ob es die Regierung zur Disziplinierung der Gewerkschaften nutzte. Behrens/Fichter/Frege (2001) fragen nach der Wirkung des Bündnisses auf die Ziele und Strategien der Gewerkschaften. Leggewie (1999) diskutiert, ob das Bündnis Vertrauensbildung gefördert hat.

Im Vergleich zur problemorientierten Politikwissenschaft, die die Bündnisliteratur dominiert, ergibt sich damit ein substanzieller Unterschied in Fragestellung und analytischer Vorgehensweise. Es zeigt sich, dass Analysen, wenn sie nicht gleich von dem vorgegebenen Interesse „Problemlösung" ausgehen, neue Fragen aufwerfen und neue Bewertungen des Bündnisses vornehmen. Es wird gefragt, welche Funktion die Akteure dem Bündnis im Verlauf der Tarifverhandlungen oder in den täglichen Auseinandersetzungen zwischen parteidemokratischen und staatlichen Akteuren zugewiesen haben. Nicht die Produktion kollektiver Problemlösungen durch das Bündnis steht dann im Mittelpunkt der Betrachtung, sondern die Formulierung und Verfolgung sozialer Interessen und die institutionelle Bearbeitung von Interessenkonflikten: Machterhaltungs-, Machtverschiebungs- und Machtverteilungsinteressen rücken in den Vordergrund.

Betrachtet man aber das Bündnis als Organisationspolitik oder im Hinblick auf seinen Beitrag zur Bearbeitung von sozialen Interessenkonflikten, so würde dies Schmitters These in Erinnerung rufen, dass Korporatismus ein Modus zur Vermittlung gesellschaftlicher Interessen darstellt (Schmitter 1974): Interessen würden durch die korporatistische Interessenvermittlung erst selbst definiert werden. Interessen wären also keineswegs vorgegeben, sondern würden ,in einem von sozialen Institutionen strukturierten Prozeß" (Streeck 1994: 12) - wie dem Bündnis für Arbeit - erst konstruiert.

So schlussfolgert Hassel (2002) aus einer Betrachtung des Ablaufs der Tarifrunde 2000 und der Vereinbarung über die Altersteilzeit im Bündnis für Arbeit, dass „der Bündnisprozess von den Verbänden der Chemieindustrie getrieben“ wurde, „die jedoch im Wesentlichen entlang ihrer Brancheninteressen denken" (Hassel 2002: 65-66). Streeck (2003b: Abstract) rekonstruiert den Ablauf des Bündnisses für Arbeit aus einem machtsoziologischen Blickwinkel, nämlich als „Geschichte von Konflikten um die Verteilung politischer Macht innerhalb des staatlichen Systems". Er hebt hervor, dass die Erneuerung der Bündnisidee 1998 ein Wahlkampfinstrument war, das sich aber als Regierungsinstrument und als Instrument zur Disziplinierung der Gewerkschaften als untauglich erwies. Mätzke (2000) geht schließlich auf den Entstehungskontext des Bündnisses ein (das gescheiterte Bündnis für Arbeit unter der Regierung Kohl sowie die darauf folgende Politikwechsel-Kampagne der Gewerkschaften und der SPD) und legt plausibel dar, dass die rot-grüne Reformstrategie - und damit auch die Absichten und Ziele, die die Regierung Schröder mit dem Bündnis verfolgte - nach dem Wahlsieg nur vage formuliert und erst im Bündnis selbst zu definieren waren.

Die Beiträge von Mätzke (2000), Hassel (2002) und Streeck (2003b) setzen einen interessanten Gegenpunkt zu den Autoren, die das Bündnis für Arbeit als einen der Problemlösung dienenden makrokorporatistischen Konzertierungsversuch betrachten. Ihre Analysen werfen die Frage nach der Wechselwirkung zwischen regierungs-, partei-, 
verbands-, sozial- und tarifpolitischem Alltagsgeschäft und dem Bündnis für Arbeit auf. Im Folgenden soll diese Perspektive anhand der Rekonstruktion der Entstehung des Bündnisses und des Konfliktes um die Rente mit 60 fortgeführt werden.

\section{Das Bündnis für Arbeit als machtorientierte Politik}

Problemorientierte Politikwissenschaft versperrt den Blick auf interessen- und machtpolitische Auseinandersetzungen, die sich fernab von komplexen Wechselbeziehungen zwischen gesellschaftlichen Problemen und politischen Entscheidungen entwickeln. Die Frage, „wie weit Politik eigentlich an gesellschaftlicher Problemlösung orientiert ist“ (Mayntz 2001: 9) ${ }^{15}$, wird in der problemorientierten Politikwissenschaft nicht gestellt.

Im Folgenden soll nun dargelegt werden, dass der Bündnisprozess von Beginn an stark von interessen- und machtpolitischen Auseinandersetzungen geprägt wurde, und die Beteiligten das Bündnis weit weniger unter Problemlösungsaspekten betrachteten, als dies in der Literatur angenommen wurde. Dass es überhaupt zu einem Bündnis für Arbeit kam, ist mit dem Scheitern des Bündnisses für Arbeit unter der Regierung Kohl im Frühjahr 1996 und aus der Dynamik des Bundestagswahlkampfes 1998 zu erklären. Die Gewerkschaften, die den ersten Bündnisversuch mit der Zwickel-Initiative vom November 1995 selbst eingeleitet hatten, stiegen aus ihm aufgrund des Konflikts um die Lohnfortzahlung und das Kündigungsrecht aus. Sie brachen mit der Regierung Kohl. ${ }^{16}$ Spätestens nachdem auch im Mai 1997 das „Bündnis Ost“ gescheitert war, engagierten sich die Gewerkschaften in der restlichen Legislaturperiode darin, gemeinsam mit der SPD die Ablösung der christliberalen Koalition einzuleiten (vgl. dazu und zum Folgenden Müller/Wilke 1999: 78-112). Auch wenn innerhalb der Gewerkschaften Unstimmigkeiten darüber auftraten, ob die Strategie der politischen Parteinahme nicht zu riskant sei und man daher nicht alle Brücken zur Union abbrechen durfte, wurde die Idee eines neuen Bündnisses für Arbeit zum Zugpferd der Sozialdemokratie für die Bundestagswahlen 1998. Mit der Ankündigung eines Bündnisses gründete die Sozialdemokratie ihren Wahlkampf auf der Polarisierung zwischen CDU und Gewerkschaften. Das Bündnis für Arbeit war damit zunächst und ausschließlich ein Bündnis zwischen Sozialdemokratie und Gewerkschaften für einen grundlegenden Regierungswechsel. Dabei ging es den Gewerkschaften auch darum, sich in der Öffentlichkeit als Meinungsführer und Reformkraft zu präsentieren. ${ }^{17}$ Verbanden Sozialdemokratie und Ge-

15 Renate Mayntz (2001) weist damit auf die Selektivität der steuerungstheoretischen Perspektive bzw. derjenigen Ansätze hin, die das Politische als Problembearbeitungsprozess konzeptualisieren.

16 So nahm selbst der DGB-Vorsitzende Dieter Schulte, der aufgrund seiner Rolle als gewerkschaftlicher Ansprechpartner der Bundesregierung eigentlich parteipolitische Zurückhaltung hätte üben müssen, Anfang 1997 ungewöhnlich deutlich für eine neue Regierungsmehrheit Stellung: „Wir brauchen eine andere Politik. Wir brauchen soziale Gerechtigkeit. Und dafür brauchen wir eine politische Mehrheit, die ich momentan nur außerhalb der derzeitigen Regierungskoalition sehe" (Schulte: Gewerkschaften werden notfalls für Rot-Grün kämpfen, dpa 0217 vom 6.1.1997; zit. nach Müller/Wilke 1999: 80).

17 Diese Bedeutung der gewerkschaftlichen Bündnis-Kampagne brachte der sonst eher moderate Vorsitzende der IG BCE Hubertus Schmoldt auf dem DGB-Kongress im Mai 1998 deutlich 
werkschaften mit dem Bündnis-Projekt deutlich ein machtpolitisches Interesse, so äuBerten sich die Arbeitgeber- und Wirtschaftsverbände, die an einen deutlichen Wahlsieg der SPD nicht glaubten und zum Teil offen die Wiederwahl der amtierenden Regierung befürworteten, zu jener Zeit noch eher skeptisch. ${ }^{18}$ Die Gewerkschaften brachten während des Wahlkampfes zudem ihre üblichen Forderungen nach Umverteilung von Arbeit und Arbeitszeit ein. ${ }^{19}$ Letzteres kann als Orientierung der Gewerkschaften an der Lösung von Beschäftigungsproblemen interpretiert werden, spricht aber ebenso für eine Orientierung der Gewerkschaften an ihrem Eigeninteresse, ihre Mitglieder vor Lohnkonkurrenz zu schützen.

Die Funktion des Bündnisses als Wahlkampfinstrument hatte zur Konsequenz, dass nach den gewonnenen Wahlen der Arbeitsauftrag des Bündnisses neu zu definieren war. In seiner Regierungserklärung vom 10. November 1998 nannte Bundeskanzler Schröder das „Bündnis für Arbeit“" sechs Mal. Er wies dem Bündnis für Arbeit vier Funktionen zu:

(1.) die „Verbesserung und Modernisierung der beruflichen Bildung und Qualifikation“ $(55(\mathrm{~A}))$,

(2.) ein „ständiges Instrument zur Bekämpfung der Arbeitslosigkeit“ (56(A)) zu sein,

(3.) eine „Vereinbarung" über „die zukunftsfähige Erneuerung der betrieblichen Altersvorsorge" $(60(\mathrm{~B}))$ zu schaffen sowie

(4.) ein „Ort zu sein, um sich [...] drängenden Fragen zu stellen: Welche Spielräume kann die Abgabenpolitik des Staates, kann die Tarifpolitik schaffen? Was bedeutet es, die Sozialleistungen stärker auf Bedürftige zu konzentrieren? Welche Spielräume schaffen wir für Investitionen, und welche Möglichkeiten bieten Instrumente wie Investivlohn und ähnliches? Welche Chancen bieten sich für uns alle, auch für die Beschäftigung, bei der Flexibilisierung der Arbeitszeiten Tarifpolitik durch die staatliche Abgabenpolitik, über die Veränderung der Sozialpolitik hin zu einer stärkeren Konzentration auf Bedürftige und über die Flexibilisierung der Arbeitszeiten?" (56(C)) (Stenographische Berichte des Deutschen Bundestages 1998: 47(D)$67(\mathrm{~B}))$.

zum Ausdruck: „Unsere Initiative für ein Bündnis für Arbeit war ein Mobilisierungserfolg ohnegleichen. Der Begriff ist heute in aller Munde, und die von uns vorgesehenen Themen stehen nach wie vor im Mittelpunkt des gemeinsamen Interesses. Wir sollten alles dafür tun, damit dies auch so bleibt" (zit. nach Müller/Wilke 1999: 120).

18 So Olaf Henkel (BDI-Präsident) auf die Frage, ob er sich mit dem damaligen SPD-Kanzlerkandidaten Gerhard Schröder zu einem Bündnis für Arbeit im Kanzleramt treffen würde: „Wir haben in Deutschland zu wenig eckige und kantige Entscheidungen" (Interview im Spiegel Nr. 1 vom 29.12.1997: 74). Der damalige Präsident von Gesamtmetall Werner Stumpfe äußerte im April 1998: „Man braucht kein Bündnis für Arbeit, damit die Regierung begreift, dass die Lohnzusatzkosten gesenkt werden müssen" (FR vom 11.04.1998: 5). Und auch noch am Vorabend des ersten Bündnistreffens hielt die Skepsis an. So beantwortete der BDA-Präsident Dieter Hundt die Frage „Was ist ein Bündnis für Arbeit“ Anfang Dezember 1998 folgendermaßen: „Ein Bündnis für Arbeit ist für mich in allererster Linie eine Regelung auf betrieblicher Ebene, in der über die zukünftige Geschäfts- und Beschäftigungsentwicklung in einem Unternehmen Aussagen getroffen werden. Da geht es zum Beispiel um die Anwendung und extensive Nutzung der Tarifverträge in der Arbeitszeitgestaltung. Solche Bündnisse für Arbeit gibt es in den Betrieben hunderttausendfach, unter anderem in meinem eigenen" (FAZ vom 2.12.1998: 18).

19 Vgl. dazu die zusammenfassende Darstellung der Diskussion über das Bündnis für Arbeit auf dem 16. DGB-Kongress im Mai 1998 in Müller/Wilke (1999: 112-124). 
Bis auf die Ankündigung, die betriebliche Altersvorsorge zu thematisieren, blieben die Äußerungen des Kanzlers über das Bündnis inhaltlich unbestimmt. Die Formulierung der Agenda überließ der Kanzler der ersten Bündnisrunde.

Auch die Frage, warum die Verbände die Einladung des Bundeskanzlers zum Bündnis für Arbeit annahmen, lässt sich auf der Grundlage von macht- und interessenpolitischen Kalkülen der Akteure beantworten. Nach der Regierungserklärung hätte eine Verweigerung der Teilnahme am Bündnis Gewerkschaften und Arbeitgeberverbände mit dem Vorwurf der Regierung belastet, ihr bei der Bekämpfung der Arbeitslosigkeit die Unterstützung zu versagen. Auf Seite der Arbeitgeber- und Wirtschaftsverbände scheint zudem ein weiteres Motiv wirksam gewesen zu sein. Vor allem in den ersten Monaten der rot-grünen Regierung sahen die Arbeitgeber- und Wirtschaftsverbände das Bündnis als Instrument, der von ihnen befürchteten roten Allianz zwischen Sozialdemokratie und Gewerkschaften Einhalt zu gebieten, ${ }^{20}$ sich ein mögliches Gegengewicht gegen die Steuer- und Finanzpolitik des Bundesfinanzministers Oskar Lafontaine zu erhalten ${ }^{21}$ und wenn möglich, „unpopuläre Maßnahmen“ durch das Bündnis zu beschließen. ${ }^{22}$ Die Arbeitgeber konnten zudem hoffen, dass das Bündnis - oder vielmehr die Bundesregierung mit Hilfe des Bündnisses - dazu beitragen würde, die Gewerkschaften zu Lohnzurückhaltung und zu einer langfristigen Tarifpolitik zu verpflichten. Bei den Gewerkschaften bestand das Interesse am Bündnis für Arbeit vor allem darin, die eigene gesellschaftspolitische Bedeutung öffentlichkeitswirksam zur Geltung zu bringen. Dies konnten sie auch nach innen gegenüber ihren Mitgliedern nutzen. Am differenziertesten gestaltete sich die Motivlage auf Seiten der Bundesregierung: So konnte der Bundeskanzler vom Bündnis vor allem vielerlei Entlastungseffekte erhoffen, unter anderem durch gemeinsame sozialpolitische Initiativen der Sozialpartner. Der Bundeskanzler konnte das Bündnis aber auch als Instrument betrachten, um das eigene Lager, die Fraktion, die Ministerien, die Traditionalisten der Partei und den Selbstverwaltungskomplex um die Bundesanstalt für Arbeit zu domestizieren (Streeck 2003b).

20 Diese Motivlage der Wirtschaft bestätigte Rogowski am 26. März 2001 in einem Interview mit Focus: Focus: „Warum gehen Sie nicht einfach raus, wenn sich bei diesen Themen [Lohnfortzahlung, Kündigungsschutz und Betriebsverfassung, TR] nichts bewegt?" Rogowski: „Wer aussteigt, verliert jede Einflussmöglichkeit. Außerdem gilt unverändert ein Satz von Herbert Wehner: Wer rausgeht, muss auch wieder reinkommen" (Focus Nr. 13 vom 26.3.2001: 274). Vgl. auch den DGB-Vorsitzenden Dieter Schulte in einem DLF-Interview vom 3. März 2001: „Und da es ja nicht nur dumme, sondern auch kluge Arbeitgeber gibt, bin ich fest davon überzeugt, dass sie nicht aussteigen werden. Denn sie würden ja eine Allianz zurücklassen zwischen Gewerkschaften und Bundesregierung. Und ich könnte mir vorstellen, dass sie uns genauso wenig trauen" (DLF 2001: 6).

21 So äußerte die BDA vor dem ersten Bündnis-Treffen: „Die BDA wird sich der Einladung zu Gesprächen nicht verschließen. Sie wird selbst die Initiative zu Gesprächen mit dem Ziel ergreifen, die von der neuen Bundesregierung angekündigten sozial-, steuer- und wirtschaftspolitischen Maßnahmen zu verhindern, da diese Wettbewerbsfähigkeit und Arbeitsplätze vernichten würden" (BDA 1998: 2).

22 So der Präsident von Gesamtmetall Werner Stumpfe im FR-Interview im April 1998. „Jawohl lasst uns ein Bündnis für Arbeit machen. Der Grund ist, dass man leichter unpopuläre Maßnahmen beschließen und durchsetzen kann, wenn man weiß, von rechts und links wird keine Kritik kommen, weil alle gleichzeitig auch ihre unpopulären Maßnahmen ergreifen werden. Als Initialzündung, denke ich, wird es so etwas wie ein Bündnis geben müssen" (FR vom 11.4.1998: 5). 
Richtig genutzt hat der Kanzler das Bündnis für Arbeit jedoch zu keinem der genannten Zwecke, ob er dies ernsthaft beabsichtigt hat, lässt sich nicht rekonstruieren. Ex post drängt sich der Schluss auf, dass das Bündnis für Arbeit aus der Sicht der Sozialdemokraten schon mit dem Wahlsieg, spätestens jedoch mit dem Abgang Lafontaines seine Schuldigkeit getan hatte.

Während also die Entstehung des Bündnisses deutlich von macht- und interessenpolitischen Kalkülen der Akteure beeinflusst wurde, gingen Regierung, Arbeitgeberverbände und Gewerkschaften bei der Formulierung der ersten gemeinsamen Bündniserklärung offenbar von konkreten beschäftigungs- und sozialpolitischen Problemen aus. So wurden dort die Bekämpfung der Arbeitslosigkeit und die nachhaltige Stärkung der Wettbewerbsfähigkeit der Wirtschaft als die wesentlichen Ziele des Bündnisses festgelegt. Unter anderem wurden eine „dauerhafte Senkung der gesetzlichen Lohnnebenkosten“, eine „strukturelle Reform der Sozialversicherung", der Abbau von Überstunden, die Förderung der Teilzeitarbeit, eine Unternehmenssteuerreform, die Flexibilisierung und Verbesserung der Möglichkeiten für das „vorzeitige Ausscheiden im Rahmen bestehender Altersgrenzen“ sowie eine "Tarifpolitik, die den Beschäftigungsaufbau unterstützt", als Wege definiert, um diese Ziele zu erreichen (Gemeinsame Erklärung vom 7. Dezember 1998: II.).

Bei einer Analyse der weiteren Bündniserklärungen wird jedoch sehr schnell deutlich, dass Staat und Verbände die Problemlösungskapazität des Bündnisses in der Folge weit weniger reflektierten. Zentrale beschäftigungs- und sozialpolitische Probleme wurden im Bündnis nicht angesprochen bzw. bewusst in dessen Agenda nicht aufgenommen. So fällt auf, dass der Ankündigung des Kanzlers vom April 1998, im Bündnis die Korrekturgesetze zu Rente, Lohnfortzahlung und Kündigungsschutz zu diskutieren, keine Umsetzung folgte. Die sozialpolitischen Reformen der neuen Regierung, wie die 630-DM-Reform, die Reform der Scheinselbstständigkeit und Teilzeitarbeit und eben auch die Korrekturgesetze wurden im Bündnis nicht behandelt. Bewertet man den weiteren Bündnisverlauf nach den verabschiedeten gemeinsamen Erklärungen, so zeigt sich, dass auch die großen sozial- und arbeitspolitischen Reformprojekte, die Rentenreform und die betriebliche Mitbestimmung im Bündnis nicht thematisiert wurden. Nach eigener Darstellung hatten die Gewerkschaften das „erfolgreich“ (Lang 2001b: 297) verhindert.

Von einer Reflexion beschäftigungspolitischer Probleme durch die Bündnisakteure zeugen hingegen wieder das dritte Treffen vom 6. Juli 1999, bei dem eine gemeinsame Erklärung von $\mathrm{BDA}$ und $\mathrm{DGB}$ zur Tarifpolitik verabschiedet wurde, und das fünfte Treffen vom 9. Januar 2000, mit dem der Streit um die Rente mit 60 zugunsten der Altersteilzeit beigelegt wurde. Die anderen Treffen erscheinen dagegen eher als medienwirksame Inszenierung: Das zweite (25. Februar 1999), vierte (12. Dezember 1999), achte (25. Januar 2002) und neunte, letzte Treffen (3. März 2003) endeten ohne gemeinsame Erklärungen. Das sechste und siebte Treffen (10. Juli 2000 und 4. März 2001) brachten Formelkompromisse über die Verbesserung von Qualifizierung, Ausbildung und Arbeitsförderung hervor. Zu sagen ist zudem, dass das Bündnis für Arbeit manchmal auch sozialpolitische Themen ansprach, die im fachpolitischen Alltagsgeschäft des Arbeits- oder des Bildungsministeriums ohnehin bearbeitet wurden (JobAqtiv-Reform, Ausbildung, Qualifizierung, Green-Card). 
Sowohl die Entstehung des Bündnisses als auch die Rekonstruktion der Spitzentreffen deuten an, dass die Handlungsorientierungen der Bündnisakteure einer empirischen Analyse nicht entzogen werden sollten. Statt über die Handlungsorientierungen Vorannahmen zu treffen, sollten sie in der Analyse des Bündnisprozesses thematisiert werden. Zahlreiche Ereignisse sprechen dafür, dass die Bündnisakteure die Problemlösungskapazität ihrer Institution weniger prominent vor Augen hatten als ihre machtpolitischen Eigeninteressen. Dieser Befund soll im Folgenden anhand einer Rekonstruktion des Konflikts um die „Rente mit 60“, welche die IG Metall im Bündnis durchsetzen wollte, weiter verdeutlicht werden.

Der Konflikt um die Rente mit 60 hat seinen Ausgangspunkt Mitte der 1990er Jahre. Zu jenem Zeitpunkt stieg der Beitragssatz der Rentenversicherung auf über 20 Prozent an, und eine weitere Erhöhung auf über 21 Prozent konnte 1997 nur durch Anhebung der Mehrwertsteuer verhindert werden. Zugleich sandte die damalige Bundesregierung mit Bundesarbeitsminister Norbert Blüm deutliche Signale an die Tarifpartner, dass sie die Möglichkeit der Frühverrentung radikal beenden wollte (Blüm 1995, 1996). ${ }^{23}$ Es wurden zunächst sowohl die Interessen der Bundesregierung als auch die der Tarifpartner bedient. Auf der einen Seite setzte die Regierung Änderungen im Arbeitsförderungs- und Rentenrecht durch, die Frührentner mit Einkommensverlust bestrafte. Auf der anderen Seite einigten sich Staat und Verbände mit dem Altersteilzeitgesetz von 1996 auf das - auf eine Initiative der chemischen Industrie zurückgehende - Altersteilzeitmodell: Die Bundesanstalt für Arbeit förderte einen frühzeitigen Ausstieg durch Altersteilzeit, wenn im Gegenzug der Arbeitsplatz wiederbesetzt wurde.

Schon damals kamen in der IG Metall Überlegungen auf, durch einen Tariffonds Kapital anzusammeln und damit die Frühverrentung mit tariflichen Finanzierungsmodellen zu erhalten. Angetrieben wurden diese Überlegungen vom damaligen Zweiten Vorsitzenden der IG Metall, Walter Riester. Bei Spitzengesprächen mit Gesamtmetall im Oktober 1996 schlug er vor, die Einrichtung eines Tariffonds auf Branchenebene verbindlich vorzuschreiben. Gemeinsam von den Tarifvertragspartnern verwaltet und finanziert aus Anteilen des Weihnachts- und Urlaubsgeldes, aus Lohnerhöhungen und Mitteln der Bundesanstalt für Arbeit sollte dieser Fonds innerhalb von fünf Jahren Kapital ansammeln, das die Tarifpartner zum Ausgleich von Einkommensverlusten bei Frühverrentung verwenden konnten. Die Rentenversicherungsbeiträge sollten durch den Fonds vollständig entrichtet werden, um so spätere Abschläge bei der Rentenzahlung zu vermeiden (BZ vom 23.10.1996: 11; FAZ vom 10.3.1997: 15). Die Umsetzung dieses Vorschlages scheiterte damals jedoch am Widerstand Gesamtmetalls, wo sich die Tariffondsidee verbandsintern nicht durchsetzen konnte, weil betriebliche Lösungen favorisiert wurden (FAZ 10.3.1997: 15). Die Großunternehmen mussten wohl auch fürchten, dass die Verpflichtung zu einem Tariffonds über die Jahre hinweg zu überhöhten Lohnabschlüssen führen würde. Darüber hinaus war das tarifpolitische Verhandlungsklima durch den Konflikt um die Lohnfortzahlung stark angeheizt, wodurch andere Themen in den Hintergrund gerieten (BZ 22.10.1996: 9).

23 Bereits in der Koalitionsvereinbarung zwischen CDU/CSU und FDP zur 13. Legislaturperiode im Herbst 1994 wurde eine Reform der Frühverrentung angemahnt: „Dem Trend der Frühverrentung älterer Arbeitnehmer muß entgegengewirkt werden“ (Koalitionsvereinbarung 1994: $35)$. 
Im Herbst 1998 gewann die Debatte um einen Tariffonds erneut Aktualität. Als gesichtswahrende Alternative zur Verkürzung der Wochenarbeitszeit, die von den Mitgliedern nunmehr abgelehnt wurde, schlug der Vorsitzende der IG Metall, Zwickel, einen Tariffonds für die Finanzierung einer Lebensarbeitszeitverkürzung vor: Zwickel drängte darauf, dass das Bündnis für Arbeit sich auf sein Konzept der „Rente mit 60“ einigen sollte (HB 10./11.10.1998: 6). ${ }^{24}$ Auch Bundesarbeitsminister Riester forderte einen Tariffonds, jedoch einen erweiterten, der zusätzlich zur Finanzierung der Altersvorsorge genutzt werden konnte (FAZ vom 2.12.1998: 18). Während die IG Metall durch das Bündnis die Bundesregierung und die Arbeitgeber für eine weitere Finanzierung der Frühverrentung gewinnen und eine gesetzliche Regelung dahingehend erreichen wollte, den Ruhestand mit 60 Jahren weiter ohne Rentenabschläge zu ermöglichen, wollte der Arbeitsminister mit seinem Tariffondsvorschlag die Gewerkschaften auf den Aufbau einer kapitalgedeckten Säule der Rentenversicherung verpflichten. Damit aber traf Riester auf den erbitterten Widerstand der IG Metall, welche die Verknüpfung des Tariffonds mit einer Reform der Altersvorsorge durch den Aufbau einer kapitalgedeckten Säule strikt ablehnte. Für Zwickel war die von Riester vorgeschlagene Kapitaldeckung eine „verkappte einseitige Beitragserhöhung“ (Zwickel 1999: 1). Wer eine dritte Säule aufbaue, ihre Finanzierung den Arbeitnehmern überlasse und gleichzeitig die Beiträge zur paritätisch finanzierten gesetzlichen Rentenversicherung senke, betreibe eine "gigantische Umverteilungsmaschine zugunsten der Unternehmer " (Zwickel 1999: 1). Die Stellvertretende DGB-Vorsitzende Engelen-Kefer nannte Riesters erweiterten Tariffonds den Beginn eines schleichenden Systemwechsels zu Lasten der gesetzlichen Rentenversicherung (FAZ vom 11.2.1999: 17).

Der Konflikt um die Rente mit 60 war in seinem Kern ein Konflikt über den zukünftigen Finanzierungsmodus der Rente. Während der Arbeitsminister aufgrund der Prognosen der Rentenversicherung über deren weitere Finanzentwicklung jede gesetzliche Rücknahme der Altersgrenzen in der Rentenversicherung ablehnte und die Finanzierungsbasis der Altersvorsorge durch einen Tariffonds und den Aufbau weiterer Säulen erweitern wollte, weigerte sich die IG Metall, für die Sicherung der Finanzen der Rentenversicherung Verantwortung zu übernehmen. Für sie bestand die Aufgabe des Bundesarbeitsministers allein darin, den Gewerkschaften die für die Frühverrentung notwendige Grundlage in der Sozialversicherung bereitzustellen. Allein zur Finanzierung der Frühverrentung war die Gewerkschaft bereit, sich an einem Tariffonds zu beteiligen. Die Regulierung und Finanzierung der Altersvorsorge trennte sie hingegen strikt von der Tarifpolitik. Deutlich brachte damals Engelen-Kefer die Skepsis mancher Gewerkschafter gegenüber Riesters erweiterten Tariffonds folgendermaßen auf den Punkt: „Die Gewerkschaften wollen die Verknüpfung von langfristigem Kapitalstock und Rente mit 60 nicht" (zit. nach FAZ vom 11.2.1999: 17).

Das Bündnis für Arbeit zeigte, dass Bundesregierung und IG Metall an der Schnittstelle von Tarif- und Sozialpolitik keine gemeinsame Perspektive für eine Reform des Wohlfahrtsstaates hatten. Es führte ferner vor Augen, dass auch unter den Einzelge-

24 Im Herbst 1999 forderte Zwickel in seiner Rede auf dem Gewerkschaftstag der IG Metall: „Wenn auch beim ,Ausstiegsmodell 60' nur halbherzig gehandelt wird, weiß ich nicht, was die Gewerkschaften über kurz oder lang noch im Bündnis für Arbeit sollen" (FAZ vom 7.10.1999: $1)$. 
werkschaften erhebliche Strategieunterschiede bestanden. So favorisierte die IG BCE die Weiterentwicklung der bereits bestehenden gesetzlichen und tariflichen Altersteilzeitregelungen und ihres eigenen Tarifvertrages zur Altersvorsorge. Im Gegensatz zur IG Metall war der Ansatz der IG BCE pragmatisch-opportunistisch: Sie nahm mit, was sie als staatliche Hilfe mitnehmen konnte (wie z.B. die Förderung der Altersteilzeit), war bemüht, Tarifvertrag und Sozialpolitik wechselseitig auf der operativen Ebene aneinander anzupassen, und unternahm es, in den folgenden Tarifrunden solche staatlich-tariflichen Lösungen durch kontinuierliche Fortentwicklung zu konsolidieren. Bereits 1996 hatte die IG BCE einen für die Beschäftigten attraktiven Altersteilzeittarifvertrag ausgehandelt. Diesen hatte sie 1998 durch einen Tarifvertrag über Altersvorsorge ergänzt, den sie in der Tarifrunde 2000 noch weiter verbessern wollte (IG BCE 1999a). Die IG BCE hielt daher während des ganzen Konfliktes um die Rente mit 60 an der Altersteilzeit fest (IG BCE 1999b). Während der IG Metall-Vorsitzende Zwickel mit einem Ausstieg aus dem Bündnis drohte, wenn die Rente mit 60 nicht umgesetzt würde (FAZ vom 27.9.1999: 1), handelte der IG BCE-Vorsitzende Schmoldt mit dem Bundesarbeitsminister die Verlängerung des Verteilzeitraums bei der Altersteilzeit aus und präsentierte in der Debatte um die Finanzierung der Rentenversicherung sein bereits mit den Arbeitgebern abgestimmtes Modell der beitragsfreien Entgeltumwandlung. In der Öffentlichkeit kritisierte Schmoldt die Ausstiegsdrohung des IG MetallVorsitzenden (BZ vom 29.9.1999: 35).

Die Arbeitgeber und Wirtschaftsverbände lehnten die Rente mit 60 von Beginn an strikt ab (vgl. Knelangen 2000). Die IG Metall scheiterte mit ihrer Forderung. Sie konnte das Bündnis nicht für ihren Tarifabschluss 2000 instrumentalisieren. Die Erklärung des fünften Bündnistreffens vom 9. Januar 2000 über „beschäftigungsorientierte und längerfristige Lohnpolitik" war das endgültige Ende der Rente mit 60 und leitete das Altersteilzeitmodell der IG BCE ein, das durch den Tarifabschluss der IG BCE über zwei Jahre Lohnzurückhaltung bei Verbesserung der Altersteilzeit besiegelt und dann auch für die IG Metall verpflichtend wurde. Im Tarifvertrag der chemischen Industrie vom 23. März 2000 wurde eine Lohnerhöhung von insgesamt 4,2 Prozent binnen 21 Monaten und in zwei Stufen vereinbart. Der Förderzeitraum der Altersteilzeit wurde von 5 auf 6 Jahre verlängert. Am 28.3.2000 kam es schließlich zum Tarifabschluss in der nordrhein-westfälischen Metallindustrie, ohne Rente mit 60 und mit einer Altersteilzeitregelung, die die IG Metall mit Lohnzurückhaltung bezahlen musste.

\section{Das Scheitern der Bündnisliteratur am Bündnis für Arbeit}

Die Politikwissenschaft hat das Bündnis für Arbeit mit wenigen Ausnahmen unter der Perspektive bewertet, ob es die Fähigkeit von Bundesregierung, Gewerkschaften und Arbeitgeberverbänden zu kollektiven Problemlösungen verbessert hat. Sie betrachtet es nach vierjähriger Laufzeit aus mehreren Gründen als gescheitert: Die Lohnnebenkosten waren höher als zuvor; der Anstieg der Arbeitslosigkeit wurde nicht gebremst; ein Beschäftigungswachstum blieb aus; auf die Lohnabschlüsse 1999 und 2002 übte das Bündnis nur wenig moderierende Wirkung aus; auch Strukturreformen des Wohl- 
fahrtsstaates wurden dort nicht beschlossen. Das Bündnis als Problemlösungsinstitution bestand seinen Test in der Praxis nicht.

Sowohl die Entstehung des Bündnisses als auch der Verlauf des Konflikts über die Rente mit 60 sprechen dafür, dass die Literatur mit ihrer Vorstellung einer Problemlösungsorientierung der Beteiligten zu kurz greift. Sie berücksichtigt zu wenig, dass der Bündnisprozess stark von konkreten interessen- und machtpolitischen Auseinandersetzungen beeinflusst wurde. Um Wirkung und Funktion des Bündnisses zu rekonstruieren, ist es erforderlich, sich die Wechselwirkungen des Bündnisprozesses mit den laufenden sozial- und tarifpolitischen Entscheidungsprozessen zu vergegenwärtigen.

Im Bündnis saßen keine Problemlöser. Vielmehr war dessen Einrichtung ein Wahlkampfinstrument. Der Konflikt um die Rente mit 60 macht deutlich, dass an der Schnittstelle von Tarif- und Sozialpolitik zwischen Staat und Gewerkschaften einerseits und unter den Gewerkschaften andererseits erhebliche Interessen- und Machtverteilungskonflikte bestanden. IG Metall und Sozialdemokratie hatten weniger Gemeinsamkeiten, als es die gemeinsame Kampagne für einen Politikwechsel während der Bundestagswahlen 1998 nach außen suggerierte. Das Bündnis für Arbeit hat dies beiden Seiten deutlich gemacht, und beide zogen daraus ihre Konsequenzen: der Bundeskanzler mit der Agenda 2010 und die IG Metall mit ihrem Konfliktkurs, der seinen Höhepunkt in dem verlorenen Streik in Ostdeutschland im Sommer 2003 erreichte. Das Ende des Bündnisses hat die Regierung gestärkt, während sich die IG Metall eines ihrer letzten Einflussinstrumente beraubt hat: Vielleicht war das Ende des Bündnisses am Ende die Problemlösung?

Um die Entstehung und Funktion des Bündnisses zu rekonstruieren, sollte machtstatt problemorientierte Politikwissenschaft der Ausgangspunkt sein, zumindest sollten jedoch die Handlungsorientierungen der Akteure reflektiert werden. Denn je nachdem welche Handlungsorientierung Akteuren unterstellt wird, lässt das Ende des Bündnisses unterschiedliche Schlussfolgerungen zu. Während eine problemorientierte Politik(wissenschaft) das Ende des Bündnisses als sein Scheitern und seinen Misserfolg betrachtet, kann eine machtorientierte Politik(wissenschaft) dessen Ende gerade als Problemlösung und Erfolg sehen. Weil die Bündnisliteratur bei der Analyse des Bündnisses die Frage nach der Handlungsorientierung der Akteure jedoch nicht gestellt, sondern Problemorientierung der Akteure vorausgesetzt hat, ist sie bei der Analyse des Bündnisses gescheitert. Die Gegenüberstellung der Bündnisliteratur mit dem Bündnis für Arbeit zeigt, wie politikwissenschaftliche Analyseergebnisse maßgeblich davon beeinflusst werden, welche Handlungsorientierungen (Präferenzen, Interessen) Akteuren unterstellt werden. Sie lehrt die Politikwissenschaft, der Frage der Formierung von Präferenzen und Interessen sowohl theoretisch als auch empirisch wieder einen höheren Stellenwert einzuräumen anstatt sie aufgrund funktionalistischer Annahmen zu Wechselbeziehungen zwischen gesellschaftlichen Problemen und politischen Entscheidungen gar nicht zu stellen.

Die politikwissenschaftliche Literatur unterscheidet sich im Übrigen in der Analyse und Bewertung des Bündnisses nicht grundsätzlich von Beiträgen, die von Mitarbeitern gewerkschaftlicher Einrichtungen, von Verbandsfunktionären oder von gewerkschaftsnahen Publizisten geschrieben wurden. Dies bestätigt die Neigung der Politikwissenschaft, das Bündnis problemlösungsorientiert zu betrachten. Die Grenzen zwi- 
schen wissenschaftlicher Rekonstruktion und politischer Konstruktion des Bündnisses erweisen sich als fließend. Der Beobachter verhält sich wie der Beobachtete. Die Politikwissenschaft täte auch aus methodologischen Gründen gut daran, der Selbststilisierung der Politiker als Problemlöser weniger zu verfallen; zumal die Beratenen den Ratschlägen der Berater sowieso nur selten folgen, sonst wäre das Bündnis ja nicht gescheitert.

\section{Literatur}

Arlt, Hans-Jürgen/Nebls, Sabine, 1999: Einleitung, in: Arlt, Hans-Jürgen/Nehls, Sabine (Hrsg.), Bündnis für Arbeit. Konstruktion, Kritik, Karriere. Opladen/Wiesbaden, 9-12.

BDA (Bundesvereinigung der Deutschen Arbeitgeberverbände), 1998: Reformen für Deutschland - Politik für mehr Wettbewerbsfähigkeit und Beschäftigung, Konzept der BDA für die 14. Legislaturperiode. Köln, 1.10.1998.

Behrens, Martin/Fichter, Michael/Frege, Carola M., 2001: Unions in Germany. Groping to Regain the Initiative. Paper for the International Seminar on „The Labour Movement: Opportunities and Strategies", 19.-21.04.2001.

Benz, Arthur, 1997: Policies als erklärende Variable in der politischen Theorie, in: Benz, Arthur/Seibel, Wolfgang (Hrsg.), Theorieentwicklung in der Politikwissenschaft: eine Zwischenbilanz. Baden-Baden, 303-322.

Bispinck, Reinhard/Schulten, Thorsten, 1999: Tarifpolitik und Bündnis für Arbeit, in: WSI-Mitteilungen 52, 870-884.

Bispinck, Reinhard/Schulten, Thorsten, 2000: Alliance for Jobs - Is Germany Following the Path of „Competitive Corporatism“? WSI-Discussion Paper Nr. 84. Düsseldorf.

Blüm, Norbert, 1995: Konkurrenzfähig bleiben, in: Bundesarbeitsblatt o. Jg., 5-12.

Bliim, Norbert, 1996: Vertrauen in die Rentenversicherung, in: Bundesarbeitsblatt o. Jg., 5-10.

Braunthal, Gerhard, 1999: The 1998 German Election. Gerhard Schröder and the Politics of the New Middle, in: German Politics and Society 17, 32-55.

BZ (Berliner Zeitung), 22.10.1996, 9.

BZ (Berliner Zeitung), 23.10.1996, 11.

BZ (Berliner Zeitung), 29.09.1999, 35.

Creutz, Helmut, 1999: Bündnis für Arbeit - Ein wichtiger Partner fehlt am Tisch, in: Zeitschrift für Sozialökonomie 36, 3-8

Detje, Richard/König, Otto, 1999: Der neue Sozialpakt, in: Sozialismus 26, 26-29.

DLF (Deutschlandfunk), 2001: ,Bündnis für Arbeit' in der Krise. Eine Sendung von Christian Hülsmeier, Redaktion: Gode Japs, ,Hintergrund Politik', 3.3.2001, 18.40-19.00; das Manuskript dieser Sendung wurde der Autorin freundlicherweise vom DLF zur Verfügung gestellt.

Dyson, Kenneth, 2001: The German Model Revisted: From Schmidt to Schröder. Unpublished Paper.

Ebbinghaus, Bernhard/Hassel, Anke, 1999: Striking Deals. The Role of Concertation in the Reform of the Welfare State. MPIfG Discussion Paper 3/1999.

Eichhorst, Werner, 2002: Bündnis für Arbeit - Chancen vergeben?, in: Sozialer Fortschritt 51, 274-281.

Esser, JosefISchroeder, Wolfgang, 1999: Neues Leben für den Rheinischen Kapitalismus. Vom Bündnis für Arbeit zum Dritten Weg, in: Blätter für deutsche und internationale Politik o. Jg., $51-61$.

Fajertag, Giuseppe/Pochet, Philippe, 1997: Social Pacts in Europe in the 1990s. Towards a European Social Pact?, in: Fajertag, Giuseppe/Pochet, Philippe (Hrsg.), Social Pacts in Europe. Brüssel, $9-25$.

FAZ (Frankfurter Allgemeine Zeitung), 10.03.1997, 15.

FAZ (Frankfurter Allgemeine Zeitung), 02.12.1998, 18. 
FAZ (Frankfurter Allgemeine Zeitung), 11.02.1999, 17.

FAZ (Frankfurter Allgemeine Zeitung), 27.09.1999, 1.

FAZ (Frankfurter Allgemeine Zeitung), 07.10.1999, 1.

Focus, 13, 26.03.2001, 274.

FR (Frankfurter Rundschau), 11.04.1998, 5.

Franz, Wolfgang, 2000: Beschäftigungsorientierte Lohnpolitik, in: Friedrich-Ebert-Stiftung (Hrsg.), Vollbeschäftigung - kein Wunschtraum. Frankfurt a.M., 50-56.

Gemeinsame Erklärung, 1998: Gemeinsame Erklärung des Bündnisses für Arbeit, Ausbildung und Wettbewerbsfähigkeit vom 7. Dezember 1998, in: http://www.buendnis-fuer-arbeit.de/05/01.html; 15.1.2001.

Haß, Hans-Joachim/Küntzel, Petra, 2003: Das Bündnis für Arbeit, Ausbildung und Wettbewerbsfähigkeit - erfolgreiches Modell der Sozialpartnerschaft?, in: Sozialer Fortschritt 52, 204-207.

Hassel, Anke, 1999: Bündnisse für Arbeit. Nationale Handlungsfähigkeit im europäischen Regimewettbewerb, MPIfG Discussion Paper 99/5.

Hassel, Anke, 2000: Soziales Kapital. Wie hoch ist der Einsatz der Tarifpartner?, in: http://www.boeckler.de/mitbestimmung/in...olltexte/Mitbestimmung-2000-06-p12.html; 3.2.2001.

Hassel, Anke, 2001: The Problem of Political Exchange in Complex Governance Systems. The Case of Germany's Alliance for Jobs, in: European Journal of Industrial Relations 7, 307-326.

Hassel, Anke, 2002: Sozialpakte. Die deutschen Gewerkschaften im Bündnis für Arbeit, in: Forschungsjournal Neue Soziale Bewegungen 15, 58-67.

Hassel, Anke, 2003: Soziale Pakte. Konzertierung als Instrument der Anpassung an die europäische Währungsunion, in: Jochem, Sven/Siegel, Nico (Hrsg.), Konzertierung in Deutschland und Europa. Das Modell Deutschland im Vergleich. Opladen, 70-104.

$H B$ (Handelsblatt), 10./11.10.1998, 6.

Heinze, Rolf G., 2003: Das „Bündnis für Arbeit“. Innovativer Konsens oder institutionelle Erstarrung?, in: Ostheim, Tobias/Egle, Christoph/Zohlnhöfer, Reimut (Hrsg.), Das rot-grüne Projekt. Eine Bilanz der Regierung Schröder 1998-2002. Wiesbaden, 137-161.

Hensche, Detlef, 1999a: Bündnis für Arbeit oder die Versuchung, Politik zu delegieren, in: Blätter für deutsche und internationale Politik o. Jg., 524-529.

Hensche, Dieter, 1999b: Feudalisierung der Politik, in: Bissinger, Manfred (Hrsg.), Konsens oder Konflikt? Wie Deutschland regiert werden soll. Hamburg, 74-79.

Hombach, Bodo, 1998: Aufbruch. Die Politik der Neuen Mitte. München/Düsseldorf.

IG BCE (Industriegewerkschaft Bergbau, Chemie und Energie), 1999a: Gemeinsames Kommuniqué. Spitzengespräch IG BCE - BAVC, Medieninformation vom 22.10.1999, in: http://www.igbce.de/IGBCE/CDA/Artikelseite/0,3898,knotenId\%3D74\%26siteId\%3D1\% 26artikelId\%3D303,00.html; 12.2.2001.

IG BCE, 1999b: Hubertus Schmoldt auf der IG-BCE-Jahrespressekonferenz, Medieninformation vom 6.12.1999, in: http://www.igbce.de/presse/medieninformation.php?w=\&nr=103070\&n=02; 25.01.2001.

Jochem, Sven/Siegel, Nico, 1999: Das Dilemma des Bündnisses für Arbeit, in: Forschungsjournal Neue Soziale Bewegungen 12, 50-60.

Jochem, Sven/Siegel, Nico, 2003: Konzertierung, Verhandlungsdemokratie und wohlfahrtsstaatliche Reformpolitik, in: Jochem, Sven/Siegel, Nico (Hrsg.), Konzertierung, Verhandlungsdemokratie und Reformpolitik im Wohlfahrtsstaat. Das Modell Deutschland im Vergleich. Opladen, 7-32.

Knelangen, Wilhelm, 2000: „Bündnis für Arbeit“ oder „Bündnis für Frührentner“? Der Streit über die Forderung nach einer „Rente mit 60“, in: Gegenwartskunde 49, 93-99.

Koalitionsvereinbarung, 1994: Das vereinte Deutschland zukunftsfähig machen. Die Koalitionsvereinbarung von CDU, CSU und FDP für die 13. Legislaturperiode des Deutschen Bundestages. 
Lang, Klaus, 2001a: Tarifautonomie und Politik. Von der Konzertierten Aktion zum Bündnis für Arbeit. Kommentar von Dr. Klaus Lang, Leiter der Abteilung 1. Vorsitzender der IG Metall zum Vortrag von Prof. Dr. Wolfgang Streeck, in: Max-Planck-Institut für Gesellschaftsforschung (Hrsg.), Die deutschen Arbeitsbeziehungen am Anfang des 21. Jahrhunderts. Eine Bestandsaufnahme. Wissenschaftliches Kolloquium im Max-Planck-Institut für Gesellschaftsforschung aus Anlass des Ausscheidens von Dr. Werner Stumpfe als Präsident von Gesamtmetall. Köln, 103108.

Lang, Klaus, 2001b: Bündnis für Arbeit - jenseits von Ausstieg und Anpassung, in: WSI-Mitteilungen 54, 294-298.

Lang, Klaus, 2002: Die Mitte gewinnen - die Mehrheit nicht verlieren. Überlegungen zur Bundestagswahl 2002, in: Forschungsjournal Neue Soziale Bewegungen 15, 49-57.

Leggewie, Claus, 1999: Böcke zu Gärtnern, in: Arlt, Hans-Jürgen/Nehls, Sabine (Hrsg.), Bündnis für Arbeit. Konstruktion - Kritik - Karriere. Opladen, 13-24.

Lehmbruch, Gerhard, 1977: Liberal Corporatism and Party Government, in: Comparative Political Studies 10, 91-26.

Lehmbruch, Gerhard, 1984: Concertation and the Structure of Corporatist Networks, in: Goldthorpe, John H. (Hrsg.), Order and Conflict in Contemporary Capitalism. Oxford, 60-80.

Lehmbruch, Gerhard, 2000: Institutionelle Schranken einer ausgehandelten Reform des Wohlfahrtsstaates. Das Bündnis für Arbeit und seine Erfolgsbedingungen, in: Czada, Roland/Wollmann, Hellmut (Hrsg.), Von der Bonner zur Berliner Republik. 10 Jahre Deutsche Einheit. Wiesbaden, 89-112.

Lowi, Theodor, 1972: Four Systems of Policy, Politics, and Choice, in: Public Administration Review 32, 398-309.

Martin, Andrew, 1997: Social Pacts: A Means for Distributing Unemployment or Achieving Full Employment?, in: Fajertag, Giuseppe/Pochet, Philippe (Hrsg.), Social Pacts in Europe. Brüssel, 37-44.

Mätzke, Margitta, 2000: Alliances for Jobs and Stability in Germany. Their Small and Implicit Effects, and the Role of Government Intentions, in: http://www.cas.northwestern.edu/cics/maetzke.pdf; 5.6.2003.

Mayntz, Renate, 2001: Zur Selektivität der steuerungstheoretischen Perspektive, MPIfG Working Paper 2001/Mai.

Müller, Hans-Peter/Wilke, Manfred, 1999: Rückkehr in die politische Arena. Die deutschen Gewerkschaften und das Bündnis für Arbeit. Bonn.

Rieble, Volker, 1999: Bündnis für Arbeit. Bedrohung oder Chance für die Tarifautonomie, in: BAVC (Hrsg.), Perspektiven. 50 Jahre Tarif- und Sozialpolitik. Heidelberg, 57-78.

Scheelhaase, Janina, 2001: Gibt es Synergieeffekte zwischen klimaschützenden Maßnahmen und dem Bündnis für Arbeit?, in: WSI-Mitteilungen 54, 706-710.

Schmitter, Philippe C., 1974: Still the Century of Corporatism?, in: Review of Politics 36, 85-131.

Schmitthenner, Horst, 1998: Bündnis für Arbeit - schon wieder?, in: Blätter für deutsche und internationale Politik o. Jg., 841-850.

Schröder, Gerhard, 1999: Das Bündnis als Fokus unserer Politik der neuen Mitte, in: Arlt, HansJürgen/Nehls, Sabine (Hrsg.), Bündnis für Arbeit. Konstruktion - Kritik - Karriere. Opladen, 49-56.

Schroeder, Wolfgang, 2001: Konzertierte Aktion und Bündnis für Arbeit. Zwei Varianten des deutschen Korporatismus, in: Zimmer, Annette/Wessels, Wolfgang (Hrsg.), Verbände und Demokratie in Deutschland. Opladen, 29-54.

Schroeder, Wolfgang, 2003: Modell Deutschland und das Bündnis für Arbeit, in: Jochem, Sven/Siegel, Nico A. (Hrsg.), Konzertierung in Deutschland und Europa. Das Modell Deutschland im Vergleich. Opladen, 107-147.

Schroeder, Wolfgang/Esser, Josef, 1999: Modell Deutschland. Von der Konzertierten Aktion zum Bündnis für Arbeit, in: Aus Politik und Zeitgeschichte B 37, 3-12.

Schulte, Dieter, 2000: Globalisierung. Ein Gestaltungsauftrag: Handlungschancen und Notwendigkeiten im Bündnis für Arbeit. Der Übersee-Club e.V. Hamburg. 
Schulte, Dieter, 2002: Nicht mit den alten Antworten kommen, in: Gewerkschaftliche Monatshefte 53, 189-195.

Siegel, Nico, 2003: Die Politische Ökonomie der Konzertierung in Deutschland. Das Beispiel Bündnis für Arbeit, in: Jochem, Sven/Siegel, Nico (Hrsg.), Konzertierung in Deutschland und Europa. Das Modell Deutschland im Vergleich. Opladen, 148-193.

Silvia, Stephen J., 1999: The Alliance for Jobs. In: German Politics and the Society 17, 84-93.

Silvia, Stephen J., 2002: The Fall and Rise of Unemployment in Germany. Is the Red-Green Government Responsible?, in: German Politics 11, 1-22.

Spiegel, Nr. 1 vom 29.12.1997, 74.

Stenographische Berichte des Deutschen Bundestages, 1998: Band 194, 14. Wahlperiode, 3. Sitzung, Dienstag, 10. November 1998, 47(D) - 67(B).

Streeck, Wolfgang, 1994: Einleitung des Herausgebers. Staat und Verbände: Neue Fragen, Neue Antworten?, in: Streeck, Wolfgang (Hrsg.), Staat und Verbände, PVS-Sonderheft 25/1994. Opladen, 7-36.

Streeck, Wolfgang, 1999a: Verbände als soziales Kapital. Von Nutzen und Nutzung des Korporatismus in einer Gesellschaft im Wandel. MPIfG Working Paper 99/2, April 1999.

Streeck, Wolfgang, 1999b: Die Gewerkschaften im Bündnis für Arbeit. MPIfG Working Paper 99/ 11, Oktober 1999.

Streeck, Wolfgang, 2001: Tarifautonomie und Politik. Von der Konzertierten Aktion zum Bündnis für Arbeit, in: Max-Planck-Institut für Gesellschaftsforschung (Hrsg.), Die deutschen Arbeitsbeziehungen am Anfang des 21. Jahrhunderts. Eine Bestandsaufnahme. Wissenschaftliches Kolloquium im Max-Planck-Institut für Gesellschaftsforschung aus Anlass des Ausscheidens von Dr. Werner Stumpfe als Präsident von Gesamtmetall. Köln, 76-102.

Streeck, Wolfgang, 2003a: From State Weakness as Strength to State Weakness as Weakness. Welfare Corporatism and the Private Use of the Public Interest. MPIfG Working Paper 03/3, March 2003.

Streeck, Wolfgang, 2003b: No longer the Century of Corporatism. Das Ende des ,Bündnisses für Arbeit'. MPIfG Working Paper 03/4, Mai 2003.

Weber, Max, 1992 [1919]: Politik als Beruf. Stuttgart.

Zwickel, Klaus, 1999: Zwickel kritisiert verkappte einseitige Beitragserhöhung in der Rentenversicherung, Pressemitteilung der IG Metall vom 17.9.1999, in: http//www.igmetall.de/aktuell/pressemitteilungen/1999/073_99.html; 1.10.2003. 